\title{
ON INTERCONNECTIONS BETWEEN THE RESEARCH OF RUSSIAN AND POLISH MATHEMATICIANS IN THE THEORY OF FUNCTIONS
}

\author{
P. L. UL'YANOV \\ Department of Mathematics and Mechanics, Moscow State University \\ Vorob'evi Gory, Moscow, Russia 119899 \\ E-mail:dyach@mail.ru
}

This symposium is dedicated to the memory of A. Rajchman (1891-1940), A. Zygmund (1900-1992) and J. Marcinkiewicz (1910-1940). They are outstanding Polish mathematicians and have gained worldwide recognition.

The scientific research of Rajchman, Zygmund and Marcinkiewicz was devoted to calculus, theory of functions of real and complex variable, functional analysis and probability theory. In their research much attention was concentrated on the metric theory of functions and its various applications.

Thus, a detailed investigation was made of trigonometric series, singular operators, approximation and interpolation theory, applications of probability theory to calculus and other questions.

It is common knowledge that Zygmund ([2], [3]) and Bari [1] published great monographs on trigonometric series, which became textbooks for many mathematicians of the world.

In the present paper we will give only a short survey of a series of results, where the research of Russian and Polish mathematicians aimed at solving common problems.

1. Uniqueness of series expansions. Let $\left\{\varphi_{n}(x)\right\}_{n=1}^{\infty}$ be a system of functions defined on $[a, b]$, and let

$$
f(x)=\sum_{n=1}^{\infty} a_{n} \varphi_{n}(x),
$$

where the series in some sense or another represents $f(x)$. The question arises: how many series of the indicated form are there or how many sequences $\left\{a_{n}\right\}_{n=1}^{\infty}$ do there exist for which (1) holds?

2000 Mathematics Subject Classification: Primary 42C25, 40A30; Secondary 42C20.

The paper is in final form and no version of it will be published elsewhere. 
This is a very important question in analysis and depends on the system $\left\{\varphi_{n}(x)\right\}$, the form of the representation in (1) and the set of classes $\{f(x)\}$.

An important case is when the representation is unique.

The first results in that direction refer to the trigonometric system and trigonometric series

$$
\frac{a_{0}}{2}+\sum_{n=1}^{\infty}\left(a_{n} \cos n x+b_{n} \sin n x\right)
$$

The partial sums of the series (2) are of the form

$$
S_{m}(x)=\frac{a_{0}}{2}+\sum_{n=1}^{m}\left(a_{n} \cos n x+b_{n} \sin n x\right)
$$

and $[0,2 \pi]=[a, b]$.

A set $E \subset(-\infty,+\infty)$ is called reduced if its derived set $E^{\prime}$ is at most countable.

In 1872 Cantor proved the following (see [4] and [1, p. 191])

THEOREM 1. If a series (2) converges to zero everywhere on $[0,2 \pi]$, then all its coefficients are zero, that is,

$$
a_{0}=a_{n}=b_{n}=0, \quad n \geq 1 .
$$

The above theorem implies that if a series (2) everywhere on $[0,2 \pi]$ converges to a finite function $f(x)$, then such a series is unique for $f(x)$. Theorem 1 was extended by Cantor to the case where (2) converges to zero on $[0,2 \pi] \backslash E$, where $E$ is a reduced set. Probably, investigating these questions Cantor conceived the idea of countable sets, and then the cardinality of sets.

Early in the $20^{\text {th }}$ century Young (see [5], [1, p. 792]) proved

THEOREM 2. Let $E$ be a countable set and suppose a series (2) converges to zero everywhere on $[0,2 \pi] \backslash E$. Then all its coefficients are zero, that is, (3) holds.

All the above leads to the following definitions. $E \subset[0,2 \pi]$ is called an $M$-set if there exists a series of the form (2) that converges to zero on $[0,2 \pi] \backslash E$ and that has nonzero coefficients. If $E$ is not an M-set, it is called a $U$-set.

Evidently, if $E \subset[0,2 \pi]$ is measurable and the measure $|E|>0$, then $E$ is an M-set. Theorem 1 and Theorem 2 suggested that if $|E|=0$, then $E$ is a U-set (see Lusin [6, pp. 249-250]). However in 1916 Menchoff (see [1, pp. 804-806], [2, p. 546] and [16, p. 31]) proved

TheOREM 3 (Menchoff [7]). There exists a perfect set $E \subset[0,2 \pi]$ with $|E|=0$ which is an $M$-set.

Then the question came up of whether there exist uncountable U-sets. A positive answer to this question was given by Rajchman [8] and Bari [10] in the early twenties. We will describe here the results of Rajchman.

In connection with these questions Rajchman came to consider H-sets introduced previously by Hardy and Littlewood (see [1, p. 732]). For these sets Rajchman gave two definitions (see [8] and [9]). $E \subset[0,2 \pi]$ is called a set of the type $\mathrm{H}$ if there exists a sequence of integers $0<n_{1}<n_{2}<\ldots$ and an interval $\Delta$ such that for every $x \in E$ and every 
$k=1,2, \ldots$ the points $n_{k} x$ do not belong to $\Delta$ modulo $2 \pi$ (see [1, pp. 732-733] and [2, pp. 498-499]). It can be noted that the closure of an H-set is an H-set. Another definition is: $E \subset[0,1]$ is called an $\mathrm{H}$-set if there exists a sequence of integers $0<n_{1}<n_{2}<\ldots$ and two numbers $\alpha \in[0,1)$ and $\delta \in[0,1)$ such that $0 \leq\left\{n_{k} x-\alpha\right\} \leq \delta$ for every $x \in E$ and $k=1,2, \ldots$, where $\{t\}$ is the fractional part of $t$ (see [1, p. 733]). If $E$ is an H-set on $[0,1]$, then $\varepsilon=\{2 \pi x: x \in E\}$ is an H-set on $[0,2 \pi]$ (see [1, p. 796]).

Every H-set is of measure zero and nowhere dense (see [1, pp. 734-735]). The following theorems hold (see [1, pp. 797 and 801]).

TheOrem 4 (Rajchman [8]). Every H-set with respect to $[0,2 \pi]$ is a U-set.

Theorem 5 (Marcinkiewicz, Zygmund [11]). Let $E \subset[0,2 \pi]$ be a $U$-set and $\theta>0$ such that

$$
E(\theta)=\{\theta x: x \in E\} \subset[0,2 \pi] .
$$

Then $E(\theta)$ is a U-set.

In the same paper Marcinkiewicz and Zygmund [11] proved that there exists a Toeplitz method for which the empty set is not a U-set for series (2) with $a_{n} \rightarrow 0, b_{n} \rightarrow 0$ as $n \rightarrow \infty$ (see also [1, p. 845]).

Until the present day no general conditions to distinguish M-sets and U-sets have been found. In this connection various sets were studied including symmetric perfect sets on $[0,2 \pi]$. To construct these sets: from the segment $[0,2 \pi]$ of length $\rho_{0}=2 \pi$ a central interval is removed and there remain two segments of equal length $\rho_{1}$; next from each of these segments central intervals of equal length are removed and there remain four segments of length $\rho_{2}$. In the $k$-th step from $2^{k-1}$ segments of equal length $\rho_{k-1}$ central intervals of equal length are removed and there remain $2^{k}$ segments of length $\rho_{k}$, etc. As a result of the process, after removing the intervals, a symmetric perfect set $E$ on $[0,2 \pi]$ is constructed. Let $\xi_{k}=\frac{\rho_{k+1}}{\rho_{k}}$. If $2^{k} \rho_{k} \rightarrow 0$ as $k \rightarrow \infty$ then $|E|=0$. It is apparent that $0<\xi_{k}<\frac{1}{2}$. Bari [12] showed that if $\xi_{k} \rightarrow \frac{1}{2}$ as $k \rightarrow \infty$, then $E$ is an M-set.

If $\xi_{k}=\frac{1}{3}$ for every $k$, then we get the Cantor set and it is an H-set, therefore by the Rajchman theorem it is a U-set.

Let $\xi_{k}=\xi \in\left(0, \frac{1}{2}\right)$ for every $k$. Then $E=E_{\xi}$ is an M-set or a U-set depending on arithmetic properties of the number $\xi$. This fact was discovered first by Bari ([13] and [12]). She proved in 1937 the following

Theorem 6 (Bari [13]). If $\xi \in\left(0, \frac{1}{2}\right)$ is a rational number, then $E=E_{\xi}$ is a $U$-set if and only if $\frac{1}{\xi}$ is an integer number, that is, if $\xi=\frac{1}{n}$ with $n=3,4, \ldots$.

For other $\xi$ we need a definition: a number $y>1$ is called a Pisot number of $n$-th degree if it is a solution of the equation $y^{n}+b_{n-1} y^{n-1}+\ldots+b_{0}=0$ with minimal $n$ and integer coefficients $\left\{b_{i}\right\}_{i=0}^{n-1}$, whereas the absolute values of the other roots of the equation are less than 1 (see [1, p. 829] and [3, p. 222]).

The sets $E_{\xi}$ have been investigated in the papers of Salem, Piatetski-Shapiro and Zygmund. In 1955 the following definitive result was obtained.

Theorem 7 (Salem, Zygmund [14]). A symmetric set $E_{\xi} \subset[0,2 \pi]$ with a constant ratio $\xi$ is a U-set if and only if $\frac{1}{\xi}$ is a Pisot number. 
The necessity was proved by Salem and the sufficiency by Piatetski-Shapiro $\left(\xi 2^{n}<1\right)$, Salem and Zygmund (see [1, p. 829] and [3, p. 227]).

As for sums of U-sets, we have ([1, p. 795])

Theorem 8 (Bari [10], [12]). A countable sum of closed U-sets is a U-set.

N. K. Bari [10] proved that every M-set contains a perfect subset and noted (see [1, p. 796]) that the union of two U-sets, one of which is closed, is a U-set, as well as, when two U-sets lie on nonoverlapping intervals, their union is a U-set. She constructed also two nonmeasurable U-sets, the sum of which coincides with $[0,2 \pi]$ and therefore is an M-set. Using this fact Zygmund [2, p. 547] constructed two measurable U-sets of measure zero, the sum of which is an M-set, and Kholshevnikova [15] gave an example of a U-set of measure zero and of the second category. She established also that the union of an arbitrary U-set and a U-set of type $F_{\sigma} \cap G_{\delta}$, as well as the union of two nonoverlapping U-sets of type $G_{\delta}$, are U-sets. These results were partly generalized by Carlet and Debs (see [16, p. 400]). In 1986 Debs and Saint Raymond [18] proved that every U-set having the Baire property is of the first category.

Recall that a set $E$ has the Baire property if there exists an open set $B$ such that $B \backslash E$ and $E \backslash B$ are of the first category. By the Kuratowski theorem this is equivalent to the fact that $E$ is the union of a $G_{\delta}$ set and a set of the first category. Since the twenties the following problem has been unsolved.

Problem. Is the union of two Borel U-sets a U-set?

This is unknown even for two U-sets of type $G_{\delta}$ (even if one of them is countable) (see [16, p. 400]).

The answer to the following question is also unknown. Let $E \subset[0,2 \pi]$ be a Borel U-set and suppose a series (2) converges to a finite function $f \in L\left(E_{1}\right)$, where $E_{1}=[0,2 \pi] \backslash E$. Is the series (2) a Fourier-Lebesgue series of $f$ ? A positive answer to this question was given by I. I. Privalov in 1923 for closed U-sets. He considered also the summation of series (2) by the Poisson method (see [1, p. 790] and [2, p. 567]). The book of Kechris and Louveau [17] describes the modern state of this theory.

Finally, we notice that problems of uniqueness of representing functions by series have been studied for various systems of functions, for example, for Walsh, Haar, Rademacher, Franklin systems, etc. (see [19], [20]).

2. Riemann theorems for function spaces. Suppose on $\Delta=[a, b]$ there is given a function series

$$
\sum_{n=1}^{\infty} \varphi_{n}(x) .
$$

If functions $\varphi_{n}(x)=a_{n} \in \mathbf{R}$ and (4) conditionally converges, then by the Riemann theorem proved in 1853 it is known that for every number $c \in \mathbf{R}$ there exists a rearrangement $\sigma(k)=\left(n_{1}, n_{2}, \ldots\right)$ of the positive integers $(1,2, \ldots)$ such that (see $[21$, p. 232])

$$
c=\sum_{k=1}^{\infty} a_{\sigma(k)}=\sum_{k=1}^{\infty} a_{n_{k}},
$$


that is, the set of sums of rearranged series is the entire line. Therefore for any series (4) in the case of $\varphi_{n}(x)=a_{n} \in \mathbf{R}$ the set of the rearranged sums is either empty, a single point, or the entire line $(-\infty,+\infty)$.

Let $K$ be a type of series convergence (or summability). It may be uniform, almost everywhere, in measure, in $L^{p}(\Delta)$, in a Banach space, summability by means of a Toeplitz method in some sense, etc.

Suppose we have a series (4) and a type $K$ of convergence. Let $S$ be the set of sums of rearranged series (4), that is, the set of functions $f$ for which there exists a rearranged series (4) that converges to $f$ in the sense of $K$.

A set $A$ is called linear if $f_{1} \in A$ and $f_{2} \in A$ implies that $\lambda f_{1}+(1-\lambda) f_{2} \in A$ for every $\lambda \in \mathbf{R}$.

Early in the $20^{\text {th }}$ century Levy and Steinitz (see [22, p. 4]) proved that the set $S$ of sums of any series consisting of $n$-dimensional vectors is linear, that is, either empty or a $k$-dimensional hyperplane with $0 \leq k \leq n$.

In 1927 Banach (see [23, p. 124]) set the problem: if a series (4) consisting of measurable functions $\varphi_{n}(x)$ for two different rearrangements converges almost everywhere to the functions $\varphi(x)$ and $\psi(x)$, does there exist for every $\lambda \in(0,1)$ a rearrangement $\sigma(k)$ such that

$$
\lambda \varphi(x)+(1-\lambda) \psi(x)=\sum_{k=1}^{\infty} \varphi_{\sigma(k)}(x)
$$

almost everywhere on $\Delta$ ? In the "Scottish Book" under N 106 Banach asked to prove that in the sense of the norm in a space $B$ for every series $\sum x_{n}$, where $x_{n} \in B$, the set $S$ of all its rearranged sums is a linear set. In the same book the negative answer was given.

TheOREM 9 (Marcinkiewicz [24, p. 188]). In $L^{2}(0,1)$ there is a series that under some rearrangements converges to $\varphi(x) \equiv 1$ and $\psi(x) \equiv 0$, but under no rearrangements converges in $L^{2}(0,1)$ to $\lambda \varphi(x)$ for any $0<\lambda<1$.

This theorem was obtained by Marcinkiewicz in the thirties. But in Marcinkiewicz's writing there is an inaccuracy in indexing functions. Besides, in the USSR this result was reported only after 1981, when the book [24] was published (see [22, pp. 44-45]).

In 1970 Nikishin proved the following

Theorem 10 (Nikishin [26]). Let $\Delta$ be $[-1,+1]$. Then there exists a series (4) of functions $\varphi_{n}(x)$ bounded on $\Delta$ such that

$$
\sum_{n=1}^{\infty}\left|\varphi_{n}(x)\right|^{2+\varepsilon} \leq C_{\varepsilon}<\infty \quad \text { for every } \varepsilon>0 \text { and every } x \in \Delta
$$

and the series (4) has two rearrangements: the first converges uniformly on $\Delta$ to $f_{1}(x) \equiv 0$ and the second to

$$
f_{2}(x)= \begin{cases}0 & \text { for } x \in[-1,0] \\ 1 & \text { for } x \in(0,1]\end{cases}
$$


Moreover, no rearrangement of the series (4) has a subsequence of partial sums that converges to the function $\lambda f_{1}+(1-\lambda) f_{2}$ for $\lambda \in(0,1)$ on any set $E \subset[-1,+1]$ with measure $|E|>1$.

It was the first negative solution in the USSR of the above Banach problems for the case of convergence almost everywhere, in measure and $L^{p}(1 \leq p<\infty)$, that is, the set of rearranged sums of the series (4) may be nonlinear. Earlier, in 1954 M. I. Kadec proved

Theorem 11 (Kadec [27]). Let $p \in[1, \infty)$ and suppose a series (4) satisfies the condition

$$
\sum_{n=1}^{\infty}\left\|\varphi_{n}\right\|_{p}^{\min (2, p)}<\infty .
$$

Then the set $S$ of rearranged sums of the series (4) in $L^{p}(0,1)$ is closed and linear.

The requirement (6) is sharp, as Nikishin ([26] for $2 \leq p<\infty)$ and Kornilov ([28] for $1 \leq p \leq 2$ ) have shown that the condition

$$
\sum_{n=1}^{\infty}\left\|\varphi_{n}\right\|_{p}^{\varepsilon+\min (2, p)}<\infty \quad \text { for every } \varepsilon>0,
$$

in general, does not assure in $L^{p}$ the linearity of the rearranged sums $S$ of the series (4).

It can be noted that from the paper of Kornilov [28] of 1980 for $p=2$ we can understand what Marcinkiewicz had in mind in solving the Banach problem in the case of $L^{2}(0,1)$. Most likely Theorem 9 belongs to Marcinkiewicz-Nikishin-Kornilov (see also [22, pp. 44-45]).

For the case of convergence almost everywhere and in measure we have

THEOREM 12 (Nikishin [25]). Suppose a series (4) satisfies

$$
\sum_{n=1}^{\infty}\left|\varphi_{n}(x)\right|^{2}<\infty \quad \text { almost everywhere on } \Delta \text {. }
$$

Then the set $S$ of all rearranged sums of the series (4) in the sense of convergence almost everywhere on $\Delta$ is linear and closed in measure.

The same holds also for convergence in measure (Nikishin [26]). The inequalities (8) and (5) provide exact conditions where the Riemann theorem holds for convergence almost everywhere and in measure, and (6) and (7) when it holds in $L^{p}(0,1)$ for $1 \leq p<\infty$.

For the space $C(\Delta)$ we have

THEOREM 13 (Kornilov [28]). Let $\omega(x)$ be a positive function on $(0, \infty)$ such that $\lim _{x \rightarrow+0} \omega(x)=0$. Then there is a series (4) of $\varphi_{n} \in C(\Delta)$ such that

$$
\sum_{n=1}^{\infty}\left\|\varphi_{n}\right\|_{C(\Delta)} \omega\left(\left\|\varphi_{n}\right\|_{C(\Delta)}\right)<\infty
$$

and, nonetheless, the set $S$ of rearranged sums, uniformly converging on $\Delta$, is not linear. 
The same conclusion holds also under the condition

$$
\sum_{n=1}^{\infty}\left|\varphi_{n}(x)\right| \omega\left(\left|\varphi_{n}(x)\right|\right)<\infty \quad \text { for every } x \in \Delta .
$$

This shows that the conditions (9) and (10) do not assure the validity of the Riemann theorem in $C(\Delta)$.

Let there be given a space $L^{p}(0,1)$ for $1 \leq p<\infty$ and a series (4). Let $S_{0}$ be the following set:

1) for $k \geq 1$ the set $S_{0}=\{0,1,2, \ldots, k\}$, that is, it consists of $k+1$ functions $f_{i}(x) \equiv i$ for $x \in[0,1]$ and $0 \leq i \leq k$;

2) $S=\{0,+1,-1,+2,-2, \ldots\}$, that is, an infinite arithmetic progression, where $f_{i}(x) \equiv i$ for $x \in[0,1]$ and $i \in \mathbf{Z}$;

3) the finite-dimensional lattice

$$
S_{0}=\left\{d_{0}(x)+\sum_{j=1}^{k} c_{j} d_{j}(x): c_{j} \in \overline{\left\{0, m_{j}\right\}} \text { or } c_{j} \in \mathbf{Z}\right\},
$$

for fixed $k \geq 1$ and $d_{j}(x) \in L^{p}(0,1)$ with $0 \leq j \leq k$.

In 1987 the following was proved:

Theorem 14 (Kornilov [29], [30]). For every $p \in[1, \infty)$ and every set $S_{0}$ there is a series (4) having the set of rearranged sums $S=S_{0}$ and (7) holds.

Similar results hold for convergence in measure [30] and in Banach spaces of infinite dimension (see [30] and [22, p. 116]). From Theorem 14 we see that the set $S$ of rearranged sums of a series (4) can be very complicated. In this connection the following problem is still unsolved.

Problem. Let $K$ be a type of convergence (or summability). For series (4) or series in Banach space there are no exact characterizations of rearranged sums $S$, that is, sets of the Riemann type.

Even for $C(\Delta)$ the definite solution is unknown. It remains unknown to me whether there exists a series (4) with $S=\left\{x, x^{2}, x^{3}\right\}$ for the case of uniform convergence.

3. Absolute convergence of Fourier series. Let $E$ be a linear set of functions $f$ defined on $[0,2 \pi]$ or $[0,1]$. In the case of $[0,2 \pi]$ we will consider $f$ to be $2 \pi$-periodic. The set $E$ is called an algebra if $f_{1} \in E$ and $f_{2} \in E$ implies $f_{1} \cdot f_{2} \in E$. A Banach space $E$ is called a Banach algebra if $f_{1} \in E$ and $f_{2} \in E$ implies

$$
\left\|f_{1} \cdot f_{2}\right\|_{E} \leq\left\|f_{1}\right\|_{E} \cdot\left\|f_{2}\right\|_{E} .
$$

Let $T=\left\{e^{i n t}\right\}_{n=-\infty}^{+\infty}$ be the trigonometric system on $[0,2 \pi]$ and $\varphi=\left\{\varphi_{n}(t)\right\}_{n=1}^{\infty}$ be an orthonormal system on $[0,1]$. Let $f \in L(0,2 \pi), \alpha \in(0,2)$ and

$$
A_{\alpha}=A_{\alpha}(T)=\left\{f: \sum_{n=-\infty}^{+\infty}\left|c_{n}(f)\right|^{\alpha}<\infty\right\}, \text { where } c_{n}=c_{n}(f)=\frac{1}{2 \pi} \int_{0}^{2 \pi} f(t) e^{-i n t} d t .
$$

In a similar manner $A_{\alpha}=A_{\alpha}(\varphi)$ for ONS $\varphi=\left\{\varphi_{n}\right\}$ are defined. 
In 1932 Wiener proved that if a continuous $2 \pi$-periodic function $f(t) \neq 0$ for every $t \in[0,2 \pi]$, then $f \in A_{1}$ implies $f^{-1} \in A_{1}$ (see [1, p. 644] and [2, p. 391]). This theorem arose from the study of Tauber theorems.

Wiener also showed that $A_{1}$ is a Banach algebra with the norm

$$
\|f\|_{B}=\sum_{n=-\infty}^{+\infty}\left|c_{n}(f)\right|<\infty .
$$

In 1934 Levy proved that if $f \in A_{1}$ and $F$ is analytic on $f([0,2 \pi])$, then $F(f) \in A_{1}$ (see [1, p. 640] and [2, p. 391]). In 1958 Katznelson established an inverse assertion to the Levy theorem: if $F$ is defined on $[A, B]$ and for every $f \in A_{1}$ with $f([0,2 \pi]) \subset[A, B]$ the function $F(f) \in A_{1}$, then $F$ is analytic on $[A, B]$ ([31, p. 102]). Therefore, the following theorem holds:

TheOREM 15 (Levy, Katznelson). Only analytic functions $F$ transform the class $A_{1}(T)$ into the class $A_{1}(T)$ (that is, $F(f) \in A_{1}$ if $f \in A_{1}$ ).

Let $\beta>0$ and $J=(a, b)$. A function $F$ is said to be in the Gevrey class $G_{\beta}(J)$ $\left(F \in G_{\beta}(J)\right)$ if on every compact $K \subset(a, b)$,

$$
\left|F^{(n)}(t)\right| \leq B^{n} n^{n \beta} \quad \text { for every } t \in K \text { and } n=0,1, \ldots,
$$

where $B=B(F, K)<\infty$. For $\beta=1$ the Gevrey class $G_{1}$ coincides with the class of analytic functions on $(a, b)$ (Pringsheim theorem) and, moreover, $G_{\beta} \supset G_{1}$ for $\beta>1$.

In 1940 the following theorem was proved (see [32, p. 66-73]).

THEOREM 16 (Marcinkiewicz). If a real function $f \in A_{\alpha}(T)$ for some $\alpha \in(0,1)$ and $F \in G_{\frac{1}{\alpha}}$ on $(a, b) \supset f([0,2 \pi])$, then $F(f) \in A_{1}(T)$.

Later Zygmund and the author have noted that in this theorem $F(f) \in A_{\alpha}(T)$, that is, $F(f) \in A_{\alpha}$ if $f \in A_{\alpha}$ and $F \in G_{\frac{1}{\alpha}}$.

In 1966 Riviere and Sagher [33] established: if a function $F$ is defined on $J=(a, b)$, $s \in(0,1]$ and if for every $f \in A_{s}$ with $f([0,2 \pi]) \subset[a, b]$ the function $F(f) \in A_{p}$ for $p<2$ ( $p$ depends on $f$ ), then $F \in G_{\frac{1}{s}}$. For the case $s=1$ this result was obtained by Helson, Kahane, Katznelson and Rudin (see [31] and [33]).

From the results of Marcinkiewicz, Riviere and Sagher it follows that if $\alpha \in(0,1)$, then only the functions $F$ from the class $G_{\frac{1}{\alpha}}$ transform the class $A_{\alpha}(T)$ into the class $A_{\alpha}(T)$ (that is, $F(f) \in A_{\alpha}$ if $f \in A_{\alpha}$ ).

We do not know the solution of the following problem. Let a modulus of continuity $\omega(\delta)$ and a sequence $\tau(n)$ define the class

$$
A_{\omega, \tau}=\left\{f: \sum_{n=-\infty}^{+\infty} \omega\left(\left|c_{n}(f)\right|\right) \tau(n)<\infty\right\} .
$$

What conditions on $F$ are necessary and sufficient for $f \in A_{\omega, \tau}$ to imply $F(f) \in A_{\omega, \tau}$ ?

In this direction general results have been obtained by the author for the Haar system $X=\left\{\chi_{m}\right\}_{m=1}^{\infty}$, where the results have quite different form (see [34]-[36]) than for the trigonometric system.

Haar proved that the system $X$ is a system of convergence almost everywhere for the class $L(0,1)$, that is, the partial sums of Fourier series with respect to the Haar system 
from $f \in L(0,1)$ converge almost everywhere in $[0,1]$. In 1928 Schauder established that $\left\{\chi_{m}\right\}_{m=1}^{\infty}$ is a basis in $L^{p}(0,1)$ with $p \in[1, \infty)$ and in 1937 Marcinkiewicz [37] showed that $\left\{\chi_{m}\right\}$ is an unconditional basis in $L^{p}(0,1)$ with $p \in(1, \infty)$.

Let $f \in L(0,1)$ and

$$
a_{m}(f)=\int_{0}^{1} f(t) \chi_{m}(t) d t
$$

be its Fourier-Haar coefficient. For a modulus of continuity $\omega(\delta)$ (with $\omega(-\delta)=\omega(\delta)$ for $\delta \in(0, \infty))$ and a sequence $\tau=\{\tau(m)\}_{m=1}^{\infty}$ with $\tau(m)>0$ we define the class of functions

$$
A_{\omega, \tau}=\left\{f: A_{\omega, \tau}(f)<\infty\right\} \quad \text { where } A_{\omega, \tau}(f)=\sum_{m=2}^{\infty} \omega\left(\left|a_{m}(f)\right|\right) \tau(m) .
$$

Let $A_{\omega}=A_{\omega, \tau}$ for $\tau(m) \equiv 1$ and $A_{\alpha}=A_{\delta^{\alpha}}$ with $0<\alpha \leq 1$.

TheOREm 17. Let $\alpha \in(0,1]$ and a finite function $F(t)$ be defined on $(-\infty,+\infty)$. Then $F(f) \in A_{\alpha}$ for every $f \in A_{\alpha}$ if and only if there is a number $D$ for which $F \in \operatorname{Lip}_{D} 1$ (that is, $|F(x)-F(y)| \leq D|x-y|$ for every $x$ and $y$ ). Under these conditions

$$
A_{\alpha}(F(f)) \leq D^{\alpha} \gamma_{\alpha} A_{\alpha}(f) \quad \text { for every } f \in A_{\alpha}, \quad \gamma_{\alpha}=\frac{2^{\frac{\alpha}{2}}}{2^{\frac{\alpha}{2}}-1} .
$$

It may be noted that in Theorem 17 for the Haar system the conditions on $F$ do not depend on $\alpha$, whereas in the trigonometric case they depend on $\alpha$ (Gevrey classes $G_{\frac{1}{\alpha}}$ ).

Theorem 18. Let $\omega=\omega(\delta)$ be a modulus of continuity. For every $F \in \operatorname{Lip}_{D} 1$ the inequality

$$
A_{\omega}(F(f)) \leq C_{\omega, F} A_{\omega}(f) \quad \text { for every } f \in L^{\infty}(0,1)
$$

holds if and only if

$$
\int_{0}^{\delta} \frac{\omega(t)}{t} d t \leq B \omega(\delta) \quad \text { for every } \delta \in(0, \infty) .
$$

This condition for $\delta \in(0,1]$ arises in 1955 in a paper of Bari. The author obtained it in 1953 but in a different form (see [38]).

To formulate general results we need a definition. A measurable and positive function $\psi(t)$ is called almost-increasing (resp. almost-decreasing) on $(a, b)$ if there exists a constant $C>0$ such that $\psi\left(t_{1}\right) \leq C \psi\left(t_{2}\right)$ (resp. $\psi\left(t_{1}\right) \geq C \psi\left(t_{2}\right)$ ) for every $t_{1}$ and $t_{2}$ from $a<t_{1}<$ $t_{2}<b$. The following theorem holds.

THEOREM 19. Let $\omega(\delta)$ be a modulus of continuity and $\tau=\tau(t)$ be an almost-increasing function on $[1, \infty)$ with $\tau(2 t) \leq C_{1} \tau(t)$ for $t \geq 1$. The inequality

$$
A_{\omega, \tau}(F(f)) \leq C_{\omega, \tau, D} A_{\omega, \tau}(f)
$$

holds for every $f \in L(0,1), F \in \operatorname{Lip}_{D} 1$ and for some constant $C_{\omega, \tau, D}$ if and only if

$$
\int_{2^{-\frac{N}{2}}}^{1} \frac{\omega(\delta t)}{t} \tau\left(2^{N} t^{2}\right) d t \leq C_{2} \tau\left(2^{N}\right) \omega(\delta) \quad \text { for every } \delta \geq 0 \text { and } N=1,2, \ldots
$$

Corollary 1. If $\tau(t)=t^{\beta}$ for $t \in[1, \infty)$ and $\beta>0$, then (12) holds for every modulus of continuity $\omega(\delta)$. 
Corollary 2. If $\tau(t)=(\ln t)^{\beta}$ for $t \in[1, \infty)$ and $\beta \geq 0$, then (12) holds if and only if (11) holds.

It can be noted that in Corollary 2 the condition (11) does not depend on $\beta \geq 0$. For decreasing functions the following theorem holds.

THEOREM 20. Let $\omega(\delta)$ be a modulus of continuity and $\tau(t)$ be an almost-decreasing function on $[1, \infty)$ with $\tau(t) \leq C_{3} \tau(2 t)$ for $t \geq 1$. The inequality (12) holds if and only if

$$
\int_{2^{-\frac{(N+1)}{2}}}^{1} \frac{\omega(\delta t)}{t} \tau\left(1+2^{N+1} t^{2}\right) d t \leq C_{4} \tau\left(2^{N}+1\right) \omega(\delta)
$$

for every $\delta \geq 0$ and $N=0,1, \ldots$.

Corollary 3. If $\tau(t)=t^{-\gamma}$ for $t \in[1, \infty)$ and $\gamma>0$, then (12) holds if and only if

$$
\delta^{2 \gamma} \int_{0}^{\delta} \frac{\omega(t)}{t^{1+2 \gamma}} d t \leq C_{5} \omega(\delta) \quad \text { for every } \delta \in(0, \infty) .
$$

Note that the condition (13) for $\omega(\delta) \not \equiv 0$ can hold only for $0 \leq \gamma<\frac{1}{2}$. For $\gamma \geq \frac{1}{2}$ the condition (13) holds only for $\omega(\delta) \equiv 0$. It can be noted that in this case the conditions depend on $\gamma$.

Now about algebras. With respect to the Haar system the set $A_{1}$ is not an algebra, as there exists $f \in A_{1}$ such that $f^{2} \notin A_{1}$. Hence some limitations are needed. Let $A_{\omega}^{(\infty)}=A_{\omega} \cap L^{\infty}$.

TheOREM 21. 1) Let $\omega(\delta)$ be a modulus of continuity. $A_{\omega}^{(\infty)}$ is an algebra and, moreover,

$$
A_{\omega}\left(f^{2}\right) \leq C_{\omega} A_{\omega}(f) \quad \text { for } f \in A_{\omega}^{(\infty)} \quad \text { with }\|f\|_{\infty} \leq 1,
$$

if and only if (11) holds for $\delta \in(0,1]$.

2) Let $\omega(\delta)$ be a modulus of continuity. $A_{\omega}^{(\infty)}$ is an algebra and

$$
A_{\omega}\left(f^{2}\right) \leq C_{\omega} A_{\omega}\left(\|f\|_{\infty} f\right) \quad \text { for every } f \in A_{\omega}^{(\infty)}
$$

if and only if (11) holds for $\delta \in(0, \infty)$.

Problem. We do not know of any result in this direction for other orthogonal systems (Walsh, Franklin, Ciesielski systems, etc.).

\section{References}

[1] N. K. Bari, Trigonometric Series, GIFML, Moscow, 1961.

[2] A. Zygmund, Trigonometric Series, Vol. 1, Mir, Moscow, 1965.

[3] A. Zygmund, Trigonometric Series, Vol. 2, Mir, Moscow, 1965.

[4] G. Cantor, Ueber die Ausdehnung eines Satzes aus der Theorie der trigonometrischen Reihen, Math. Ann. 3 (1872), 123-132.

[5] W. H. Young, A note on trigonometrical series, Messenger of Math. 38 (1909), 44-48.

[6] N. N. Luzin, Integral and Trigonometric Series, GIFML, Moscow-Leningrad, 1951.

[7] D. E. Menshoff, Sur l'unicité du développement trigonométrique, C. R. Paris 163 (1916), 433-436. 
[8] A. Rajchman, Sur l'unicité du développement trigonométrique, Fund. Math. 3 (1922), 287-301; 4 (1923), 366-367.

[9] A. Rajchman, Sur la multiplication des séries trigonométriques et sur une classe d'ensembles fermés, Math. Ann. 95 (1926), 388-408.

[10] N. K. Bari, Sur l'unicité du développement trigonométrique, C. R. Paris 177 (1923), 1195-1197; Fund. Math. 9 (1927), 62-118.

[11] J. Marcinkiewicz and A. Zygmund, Two theorems on trigonometric series, Matem. Sbornik 2 (44) (1937), 733-738.

[12] N. K. Bari, The problem of the uniqueness of the expansion of a function into trigonometric series, Uspekhi Matem. Nauk 4 (1949), no. 3, 3-68.

[13] N. K. Bari, Sur le rôle des lois diophantiques dans le problème d'unicité du développement trigonométrique, Matem. Sbornik 2 (44) (1937), 699-722.

[14] R. Salem and A. Zygmund, Sur un théorème de Piatetcki-Shapiro, C. R. Paris 240 (1955), 2040-2042.

[15] N. N. Kholsevnikova, On a union of less than continuum many closed U-sets, Vestnik MGU Matem Mech. 1981, no. 1, 51-55.

[16] D. E. Menshoff, Selected Papers, Mathematics, Nauka, Moscow, 1997.

[17] A. Kechris and A. Louveau, Descriptive Set Theory and the Structure of Sets of Uniqueness, London Math. Soc. Lecture Note Series 128, Cambridge Univ. Press, 1989.

[18] G. Debs and J. Saint Raymond, Ensembles boréliens d'unicité et d'unicité au sens large, Ann. Inst. Fourier (Grenoble) 37 (1987), 217-239.

[19] V. A. Skvortsov, Questions of uniqueness of expansions of functions into series with respect to Haar and Walsh systems and generalized integrals, self-report of the doctoral thesis, MGU, 1982.

[20] G. G. Gevorkyan, Questions of convergence and uniqueness of trigonometric series and Franklin series, self-report of the doctoral thesis, MIAN, 1991.

[21] B. Riemann, On the Possibility of Representation of Functions via Trigonometric Series, GITTL, Moscow-Leningrad, 1948.

[22] V. M. Kadec and M. I. Kadec, Rearrangements of Series in Banach Spaces, Tartu, 1988.

[23] W. Orlicz, Über die unabhängig von der Anordnung fast überall konvergenten Funktionenreihen, Bull Acad. Polonaise 1927, 117-125.

[24] The Scottish Book, Birkhäuser, Boston, 1981.

[25] E. M. Nikishin, On a set of sums of function series, Matem. Zametki 7 (1970), 403-410.

[26] E. M. Nikishin, Rearrangements of function series, Matem. Sbornik 85 (1971), 272-286.

[27] M. I. Kadec, On the conditionally convergent series in $L_{p}(0,1)$, Uspekhi Matem. Nauk 9 (1954), 107-109.

[28] P. A. Kornilov, On the rearrangements of conditionally convergent function series, Matem. Sbornik 113 (1980), 598-616.

[29] P. A. Kornilov, On the structure of the set of sums of function series, Vestnik MGU Matem. Mech. 1988, no. 4, 9-13.

[30] P. A. Kornilov, On the set of sums of conditionally convergent function series, Matem. Sbornik 137 (1988), 114-127.

[31] J.-P. Kahane, Absolutely Convergent Fourier Series, Mir, Moscow, 1976.

[32] J. Marcinkiewicz, Sur la convergence absolue des séries de Fourier, Mathematica (Cluj) 16 (1940), 66-73.

[33] N. M. Riviere and Y. Sagher, The converse of Wiener-Levi-Marcinkiewicz theorem, Studia Math. 28 (1966), 133-138. 
[34] P. L. Ul'yanov, On one algebra of functions, Acta Sci. Math. 49 (1985), 169-178.

[35] P. L. Ul'yanov, The superposition of functions and Fourier coefficients, Trudy MIAN 172 (1985), 338-348.

[36] P. L. Ul'yanov, DAN 262 (1982), 542-545; 269 (1983), 1054-1056; 333 (1993), 157-160; 350 (1996), 25-28; 355 (1997), 612-615; 361 (1998), 31-34.

[37] J. Marcinkiewicz, Quelques théorèmes sur les séries orthogonales, Ann. Soc. Polon. Math. 16 (1937), 84-96.

[38] P. L. Ul'yanov, On the moduli of continuity and Fourier coefficients, Vestnik MGU Ser. 1 1995, no. 1, 37-52. 